\title{
LÓGICA, ORDEM E SISTEMAS IMPLICATIVOS
}

Logic, order and implicative systems

Lógica, ordem y sistemas implicativos

Cristiane Alexandra Lázaro'

Universidade Estadual Paulista 'Júlio de Mesquita Filho, Bauru, SP, Brasil.

Hércules de Araujo Feitosa ${ }^{2}$

Universidade Estadual Paulista 'Júlio de Mesquita Filho, Bauru, SP, Brasil.

Marcelo Reicher Soares ${ }^{3}$

Universidade Estadual Paulista 'Júlio de Mesquita Filho', Bauru, SP, Brasil.

\section{Resumo}

Neste artigo, tratamos de três tópicos básicos para o entendimento de uma noção de razão, a saber, relação de consequência, relação de ordem e sistema formal. A partir de uma caracterização de sistema formal, definimos as duas relações mencionadas. Destacamos uma inter-relação entre consequência e ordem. Então, apresentamos outros sistemas formais que consideram especificamente um conceito de implicação, dado pelas álgebras implicativas e pela lógica implicativa. Esta lógica implicativa, que

1 Doutora em Matemática pela Universidade Estadual de Campinas (UNICAMP), Campinas (SP), Brasil. Professora Assistente na Universidade Estadual Paulista (UNESP), Bauru (SP), Brasil. https://orcid.org/0000-0002-0260-6976.E-mail cristiane.lazaro@unesp.br

2 Doutor em Filosofia pela Universidade Estadual de Campinas, Brasil (UNICAMP), Campinas (SP), Brasil. Professor Assistente na Universidade Estadual Paulista (UNESP), Bauru (SP), Brasil. https://orcid.org/0000-0003-0023-4192. E-mail: hercules.feitosa@unesp.br

3 Doutor em Matemática pela Universidade de São Paulo (USP), São Paulo, (SP), Brasil. Professor Assistente na Universidade Estadual Paulista (UNESP), Bauru (SP), Brasil. https:// orcid.org/0000-0002-1996-5350.E-mail: reicher.soares@unesp.br 
tem como modelo as álgebras implicativas, apresenta apenas um operador, o de implicação; e este conceito de implicação, mais uma vez, remete para as noções de consequência e ordem. Por fim, a partir da caracterização de consequência que está no texto, introduzimos um modelo algébrico para esta lógica implicativa.

Palavras-chave: Consequência lógica. Ordem. Implicação. Álgebra implicativa. Lógica implicativa.

\begin{abstract}
In this paper, we discuss three basic topics for the understanding of a notion of reason, they are, consequence relation, order relation and formal system. From a characterization of formal system, we define both mentioned relations. We detach an interrelationship between consequence and order. This way we present other formal systems that indicate specifically a concept of implication, given by implicative algebras and implicative logic. This implicative logic, which has as its models the implicative algebras, has only one operator, the implication operator, that once again leads us for the consequence and order notions. Finally, from the consequence notion presented in the text, we introduce an algebraic model for this implicative logic.
\end{abstract}

Keywords: Logical consequence. Order. Implication. Implicative algebra. Implicative logic.

\title{
Resumen
}

En este artículo, tratamos tres temas básicos para entender una noción de razón, a saber, la relación de consecuencia, la relación de orden y el sistema formal. A partir de una caracterización de sistema formal, definimos las dos relaciones mencionadas. Destacamos una interrelación entre la consecuencia y el orden. Luego, presentamos otros sistemas formales que consideran específicamente un concepto de implicación, dado por las álgebras implicativas y por la lógica implicativa. Esta lógica implicativa, que tiene como modelo las álgebras implicativas, presenta un solo operador, de la implicación; y este concepto de implicación, una vez más, se refiere a las nociones de consecuencia y orden. Finalmente, a partir de la caracterización de la consecuencia que está en el texto, introdujimos un modelo algebrico para esta lógica implicativa.

Palabras clave: Consecuencia lógica, Orden. Implicación. Álgebra implicativa. Lógica implicativa. 


\section{Introdução}

Uma noção essencial da Lógica é a consequência lógica, que investiga sob que condições um dado pode ser obtido de uma coleção de dados. De um modo simples, consideramos os dados ou informações iniciais como premissas e aquele obtido como a conclusão. Naturalmente temos uma relação de ordem nesta noção de consequência, as premissas, que são anteriores, e a conclusão que é posterior.

Contudo, a noção de consequência e a de ordem são imbricadas e nestas notas buscamos explicitar algumas destas muitas conexões. Procuramos desenvolver esta análise usando aspectos formais que fundamentam estas noções.

Temos, assim, três conceitos fundamentais para o entendimento e discussão de uma noção de razão, que são: consequência, ordem e formalização, os quais procuramos inter-relacionar, neste artigo. Tais tópicos, além de filosoficamente relevantes, são bastante básicos. As correlações entre eles permitem que a clareza eventualmente revelada em um tópico possa ser partilhada ou destacada em outro.

Iniciamos com uma apresentação bem geral de sistema formal, como usualmente tratada em textos de Lógica. Seguimos com uma apresentação algébrica do conceito de ordem.

Noutra seção, abordamos noções para a formalização do conceito de consequência lógica. Neste ponto já podemos inter-relacionar os tópicos de consequência e ordem.

Nos passos seguintes, introduzimos, com bastante detalhes, uma lógica que conta apenas com o operador de implicação. Provas originais são apresentadas, para alguns resultados nesta lógica proposicional.

Finalmente, mostramos como podemos usar os espaços de consequência para gerar um modelo para esta lógica, a qual por enfatizar a implicação, de algum modo, realça a ordem dada no 'se, então' e a consequência lógica. 


\section{Sistemas formais}

As definições do que é a Matemática ou do que é a Lógica, ainda nos dias de hoje, apresentam aspectos controversos e não é nosso objetivo abordar estas questões neste trabalho. Nossos esforços estarão concentrados em estudar diversos tipos particulares do que chamamos de Sistemas Formais e em buscar estabelecer relações entre eles. Isto é, apresentaremos uma caracterização do conceito de sistema formal e tudo que seguirá serão instanciações dessa abstração, bem como as relações que conseguirmos estabelecer entre as mesmas.

A noção de sistema formal é tão eficaz que muitas disciplinas tentam obter para si uma abordagem desse tipo. Ciências como a Física e a Química, não se baseiam integralmente em sistemas formais, mas em um sistema semiformal, que apenas evoca aspectos da formalização apresentada pela Matemática.

O primeiro item a compor um sistema formal contemporâneo $S$ é sua linguagem. Ela fica determinada ao se explicitar o seu conjunto de símbolos, algumas vezes chamado de alfabeto, o qual denotamos aqui por $\mathbf{A}$.

$\mathrm{Na}$ busca por clareza, precisão e objetividade, trocamos a linguagem natural por linguagens artificiais ou formais.

O conjunto das expressões geradas a partir do alfabeto $\mathbf{A}$ é o conjunto de todas as sequências finitas de símbolos de $\mathbf{A}$ ou justaposições finitas de símbolos de $\mathbf{A}$.

Para a distinção das expressões bem formadas, obtidas a partir do alfabeto e que constituirão a linguagem deste sistema formal, temos regras gramaticais precisas.

Dentre estas expressões bem formadas, destacamos o conjunto das fórmulas, o qual denotamos por $\mathbf{F}$. Dependendo do sistema formal considerado, podemos ter outras classes de expressões bem formadas. Indicamos as fórmulas por letras gregas minúsculas: $\varphi, \psi, \sigma$.

Outro item que pode ocorrer nos sistemas formais é um conjunto de axiomas ou postulados, o qual denotaremos por P. Neste caso, o sistema 
formal é chamado de sistema formal axiomático. O conjunto dos axiomas é subconjunto do conjunto das fórmulas. Assim, o que representa as leis do sistema axiomático são as fórmulas, e estas serão os portadores de verdade, aqueles elementos que serão tomados como verdadeiros ou falsos.

É possível desenvolver sistemas formais que não sejam axiomáticos. Neste caso, embora o conjunto $\mathbf{P}$ seja vazio, ainda assim, é possível termos uma relação de consequência muito precisa. São exemplos de tais sistemas formais os tablôs, os sistemas de dedução natural e alguns cálculos de sequentes.

O último componente de um sistema formal é um conjunto $\mathbf{R}$ das regras de dedução. Não há sistema formal sem regras, isto é, sempre temos $\mathbf{R} \neq \varnothing$. São estas regras que possibilitam a dedução, ou demonstração, no sistema formal. Assim, a passagem de uma situação para outra situação é regida pelas regras, que explicitam a noção de dedução.

Em um sistema axiomático $S$, chamamos de teorema cada fórmula $\varphi$ que:

(i) é um dos axiomas de $\mathbf{S}$; ou

(ii) é conclusão de uma regra de dedução de $\mathbf{R}$ em que as hipóteses são teoremas de $\mathbf{S}$.

Em um sistema formal axiomático $\mathbf{S}$ os conceitos de dedução e demonstração são muito precisos e inequívocos. Eles caracterizam precisamente o que chamamos de consequência sintática ou consequência dedutiva.

Uma demonstração em $\mathbf{S}$ é uma sequência $\varphi_{1}, \varphi_{2}, \ldots, \varphi_{\mathrm{n}}$ de fórmulas tais que, para cada $\mathrm{i}, 1 \leq \mathrm{i} \leq \mathrm{n}, \varphi_{\mathrm{i}}$ é um axioma ou $\varphi_{\mathrm{i}}$ é deduzida de fórmulas precedentes por alguma regra $\mathrm{R}$ de $\mathbf{R}$. Um teorema de $\mathbf{S}$ é como chamamos a última fórmula $\varphi_{\mathrm{n}}$ de uma demonstração. A fórmula $\varphi_{\mathrm{n}}$ é o teorema e o procedimento é uma demonstração de $\varphi_{n}$ em $\mathbf{S}$.

Segue destas definições que uma demonstração é um procedimento e é finito, pois acaba no passo $n$.

O conceito de dedução é uma leve ampliação do conceito de demonstração, de modo que toda demonstração é um caso de dedução.

Uma fórmula $\psi$ é deduzida ou derivada em $\mathbf{S}$ de um conjunto $\Gamma$ de fórmulas, o que é denotado por $\Gamma \vdash \psi$, se existe uma sequência $\varphi_{1}, \varphi_{2}, \ldots$, $\varphi_{\mathrm{n}}$ de fórmulas de maneira que para cada $1 \leq \mathrm{i} \leq \mathrm{n}, \varphi_{\mathrm{i}}$ é um axioma, ou $\varphi_{\mathrm{i}} \in$ 
$\Gamma$, ou $\varphi_{\mathrm{i}}$ é deduzido de fórmulas que ocorrem anteriormente na sequência, e $\varphi_{n}$ é $\psi$. Esta sequência é uma dedução de $\psi$ a partir de $\Gamma$. Os membros de $\Gamma$ são as premissas ou hipóteses e $\psi$ é a conclusão da dedução.

Uma fórmula $\psi$ deduzida do conjunto vazio, isto é, $\varnothing \vdash \psi$, é um teorema de $\mathbf{S}$ e é usualmente denotado apenas por $\vdash \psi$.

Observamos que esta caracterização da dedução formal explica completamente a inferência dedutiva. Mais do que uma inferência, este é um argumento dedutivo, pois cada passo da dedução precisa estar muito bem justificado. Ele preserva os aspectos de necessidade, formalismo e a prioridade.

No cotidiano da matemática usual, não somos tão explícitos nas deduções e demonstrações. O texto fica menos pedante, mais breve, embora menos preciso, perfeitamente compreensível pelos praticantes. Se fizermos questão da precisão, teremos que pagar o preço da prolixidade.

\section{Sobre o conceito de ordem}

O conceito de ordem ou ordenação é essencial para a Lógica e, também, para a Matemática, que historicamente procurou formalizar o conceito no contexto das relações de ordem.

Veremos, nesta seção, como a Matemática na subárea Álgebra trata deste tópico.

Definição 2.1: Uma relação binária క sobre um conjunto A é uma ordem parcial se é reflexiva, antissimétrica e transitiva, isto é, valem:

(i) para todo $a \in \mathrm{A}, a \leq a$

(ii) para todos $a, b \in \mathrm{A}$, se $a \leq b$ e $b \leq a$, então $a=b$

(iii) para todos $a, b, c \in \mathrm{A}$, se $a \leq b$ e $b \leq c$, então $a \leq c$.

Definição 2.2: Um conjunto parcialmente ordenado (poset) é um par orde$\operatorname{nado}(A, \leq)$, em que $A$ é um conjunto não vazio e sé uma ordem parcial sobre $A$. Uma relação de ordem parcial é, muitas vezes, chamada apenas de relação de ordem. 
Definição 2.3: $A$ ordem $\leq$ em $A$ é uma ordem total (ou ordem linear) quando para todo par de elementos $\mathrm{x}, \mathrm{y} \in \mathrm{A}$, tem-se $\mathrm{x} \leq \mathrm{y}$ ou $\mathrm{y} \leq \mathrm{x}$.

Neste caso, temos uma estrutura de ordem total $(A, \leq)$, ou cadeia, e dizemos que $A$ é um conjunto totalmente ordenado por $\leq$. Observemos que cada ordem total é ainda uma ordem parcial.

Definição 2.4: Sejam $(A, \leq)$ um poset e $x, y \in A$. Dizemos que o elemento $x$ é estritamente menor que $y$, o que é denotado por $x<y$, quando: $\mathrm{x} \leq \mathrm{y}$ e $\mathrm{x} \neq \mathrm{y}$.

Neste caso, dizemos que a relação definida por < é uma ordem estrita. Essa relação é antissimétrica e transitiva, mas não é reflexiva.

Proposição 2.5: Um par ( $\mathrm{A}, \leq)$ é uma estrutura de ordem total se, e somente se, vale a lei da tricotomia, isto é, para quaisquer $\mathrm{x}, \mathrm{y} \in \mathrm{A}$, vale exatamente uma das condições seguintes:

$$
x<y \text { ou } x=y \text { ou } y<x .
$$

Definição 2.6: Sejam $(E, \leq)$ um poset e $\varnothing \neq A \subseteq E$. Um elemento $s$ de $E$ é um limitante superior de $A$ quando: $\forall x(x \in A \rightarrow x \leq s)$. Um elemento $i$ de $E$ é um limitante inferior de $A$ quando: $\forall x(x \in A \rightarrow i \leq x)$.

Os elementos s e $i$ não precisam estar em $A$, mas apenas em $E$.

Definição 2.7: Sejam $(E, \leq)$ um poset e $\varnothing \neq A \subseteq E$. Um elemento $M$ de $A$ é um máximo de $A$ quando: $\forall x(x \in A \rightarrow x \leq M)$. Um elemento $m$ de $A$ é um mínimo de $A$ quando: $\forall x(x \in A \rightarrow m \leq x)$.

Nestes casos, $M$ e $m$ estão necessariamente em $A$.

Segue da definição que todo máximo (respectivamente mínimo) é um limitante superior (respectivamente inferior). 
Definição 2.8: Sejam $(E, \leq)$ um poset e $\varnothing \neq A \subseteq E$. O supremo de $A$, caso exista, é o menor dos limitantes superiores de A. O ínfimo de A, caso exista, é o maior dos limitantes inferiores de $A$.

Usualmente, denotamos o supremo de $\{x, y\}$ por $\sup \{x, y\}$ e o ínfimo de $\{x, y\}$ por $\inf \{x, y\}$. O supremo de $\{x, y\}$ é o menor limitante superior de $\{x, y\}$ e o ínfimo de $\{x, y\}$ é o maior limitante inferior de $\{x, y\}$.

Se A tem um máximo (respectivamente mínimo) então este elemento é o supremo (respectivamente ínfimo).

O supremo e o ínfimo de A, caso existam, são únicos. Indicamo-los $\operatorname{por} \sup (A)$ e $\inf (A)$.

Definição 2.9: Sejam $(E, \leq)$ um poset e $\varnothing \neq A \subseteq E$. Um elemento $L \in$ $A$ é um maximal de $A$ quando: $(\forall x \in A)(L \leq x \rightarrow x=L)$. Um elemento I $\in A$ é um minimal de $A$ quando: $(\forall x \in A)(x \leq I \rightarrow I=x)$.

Para um poset $(E, \leq)$ em que $\leq$ não é total, a definição de maximal de $A$ diz que não há, em $A$, maior elemento que ele, mas não que ele é o maior elemento de A. Ou seja, podemos ter dois ou mais maximais que, eventualmente, não sejam comparáveis segundo a ordem $\leq$. Assim, este elemento, caso exista, não precisa ser único. Certamente, se há um máximo, então este é também um maximal.

Quando a relação s é de ordem total em E então, se um conjunto não vazio $A \subseteq E$ tem algum elemento maximal, esse maximal é único. Nesse caso, da ordem total, decorre que o maximal de A é também o máximo de A.

Proposição 2.10: (Lema de Zorn) Seja ( $\mathrm{A}, \leq$ ) um conjunto ordenado. Se toda cadeia em $(A, \leq)$ tem um limitante superior, então $(A, \leq)$ tem um elemento maximal.

Demonstração: Ver Feitosa, Nascimento e Alfonso (2011).

O Lema de Zorn é equivalente ao Axioma da Escolha da Teoria dos Conjuntos e introduz aspecto não construtivo na teoria em que é utilizado. Precisaremos dele para a prova de completude que vem mais à frente. 
A seguir veremos mais alguns detalhes sobre as ordens envolvidas com o conceito de Conexão de Galois. Detalhes podem ser encontrados em Feitosa, Lázaro e Nascimento (2018).

Definição 2.11:Seja $f:(A, \leq) \rightarrow(P, \leq)$ uma função entre dois conjuntos parcialmente ordenados. Então:

(i) a função f preserva as ordens se $a \leq b \Rightarrow \mathrm{f}(a) \leq \mathrm{f}(b)$;

(ii) a função f inverte as ordens se $a \leq b \Rightarrow \mathrm{f}(b) \leq \mathrm{f}(a)$.

Definição 2.12:Se f: $(A, \leq) \rightarrow(A, \leq)$ é uma função sobre o mesmo conjunto parcialmente ordenado, então:

(i) a função f é idempotente se fof $=f$;

(ii) a função fé extensiva ou inflacionária se para todo $a \in \mathrm{A}, a \leq \mathrm{f}(a)$;

(iii) a função fé deflacionária se para todo $a \in \mathrm{A}, \mathrm{f}(a) \leq a$.

Definição 2.13: Se f: $(A, \leq) \rightarrow(A, \leq)$ é uma função sobre o mesmo conjunto parcialmente ordenado, então:

(i) a função fé um operador de Tarski (operador do fecho dedutivo) se f é inflacionária, preserva ordens e é idempotente;

(ii) a função fé um operador de interior se fé deflacionária, preserva ordens e é idempotente.

Definição 2.14: Para os conjuntos parcialmente ordenados $(A, \leq)$ e $(P$, $\leq)$ e as funções $\mathrm{f}: \mathrm{A} \rightarrow \mathrm{P}$ e $\mathrm{g}: \mathrm{P} \rightarrow \mathrm{A}$, o par $(\mathrm{f}, \mathrm{g})$ é uma conexão de Galois se, para todo $a \in \mathrm{A}$ e todo $p \in \mathrm{P}$, temos:

$$
\mathrm{a} \leq \mathrm{g}(p) \Leftrightarrow p \leq \mathrm{f}(a) .
$$

Segue desta definição que se ( $f, g)$ é uma conexão de Galois, então o par $(g, f)$ é também uma conexão de Galois.

A proposição seguinte nos dá uma caracterização bastante útil para uma conexão de Galois. 
Proposição 2.15: Sejam $(A, \leq)$ e $(P, \leq)$ duas ordens parciais, $f: A \rightarrow P$ e g: $\mathrm{P} \rightarrow \mathrm{A}$ funções, $a, b \in \mathrm{A}$ e $p, q \in \mathrm{P}$. Então, o par $(\mathrm{f}, \mathrm{g})$ é uma conexão de Galois se, e somente se, valem as condições:

(i) $a \leq b \Rightarrow \mathrm{f}(b) \leq \mathrm{f}(a)$

(ii) $p \leq q \Rightarrow g(q) \leq g(p)$

(iii) $a \leq \mathrm{g}(\mathrm{f}(a))$

(iv) $p \leq \mathrm{f}(\mathrm{g}(p))$.

Assim, em uma conexão de Galois (f, $g$ ), as funções $f$ e $g$ invertem as ordens e são inflacionárias.

Proposição 2.16: Se (f, g) é uma conexão de Galois segundo as ordens parciais $(A, \leq)$ e $(P, \leq)$, então segue que $f(a)=f(g(f(a)))$ e $g(p)=g(f(g(p)))$.

Proposição 2.17: Se $\left(f, g_{1}\right)$ e $\left(f, g_{2}\right)$ são conexões de Galois segundo as ordens $(A, \leq)$ e $(P, \leq)$, então: $g_{1}=g_{2}$. Se $\left(f_{1}, g\right)$ e $\left(f_{2}, g\right)$ são conexões de Galois para $(A, \leq)$ e $(P, \leq)$, então $f_{1}=f_{2}$.

Proposição 2.18: Se o par $(f, g)$ é uma conexão de Galois para $(A, \leq)$ e $(P, \leq)$, então valem:

(i) $a \in g(P) \Leftrightarrow g(f(a))=a$

(ii) $p \in f(A) \Leftrightarrow f(g(p))=p$

(iii) $f(A)=f(g(P))$

(iv) $g(P)=g(f(A))$.

Proposição 2.19: Se o par ( $f, g)$ é uma conexão de Galois para $(A, \leq)$ e $(P, \leq)$, então:

(i) $f(a)=\max \{p \in P: a \leq g(p)\}$

(ii) $g(p)=\max \{a \in A: p \leq f(a)\}$. 


\section{Formalização conjuntista da consequência e espaços de Tarski}

Não formalizaremos, neste momento, o conceito de Lógica, apenas destacaremos neste tipo de sistema formal um de seus aspectos mais relevantes, a saber, o conceito de consequência. Nos textos de lógica, com frequência, associamos o conceito de consequência a uma relação que atribui a cada conjunto de premissas exatamente um elemento como consequência, a conclusão.

Esta abordagem generaliza a nossa concepção de inferência.

Definição 3.1: Denominamos uma relação $\mathrm{R} \subseteq \mathrm{E} \times \mathrm{E}$ de equipotente; $\mathrm{e}$ uma relação $\mathrm{R} \subseteq \mathcal{P} E) \times E$ de não equipotente, em que $\mathcal{P}(E)$ é o conjunto das partes de $\mathrm{E}$.

Como veremos a seguir, uma relação de consequência de conclusão unitária é um caso de relação não equipotente. Já uma relação de consequência de múltiplas conclusões é um exemplo de relação equipotente. Outro exemplo significativo de relação equipotente é o conceito de operador de consequência de Tarski, que também será definido a seguir.

Definição 3.2: Seja E um conjunto não vazio. Para todos $A \cup B \cup\{x, y\} \subseteq$ $\mathrm{E}$, a relação não equipotente $\vdash \subseteq \mathcal{P}(\mathrm{E}) \times \mathrm{E}$ é uma relação de consequência unitária sobre $\mathrm{E}$ se são válidas as seguintes condições:

(R1) $\{x\} \vdash x$

(R2) $A \vdash x \Rightarrow A \cup B \vdash x$

(R3) $A \vdash x$ e $A \cup\{x\} \vdash y \Rightarrow A \vdash y$.

Como neste trabalho só trataremos de relações de consequência unitária sobre $E$, nos referiremos a esta, simplesmente, como relação de consequência sobre $E$. Denotaremos o sistema dedutivo determinado pelo conjunto $E$ e pela relação de consequência $\vdash$, sobre $E$, por $(E, \vdash)$.

Definição 3.3: Seja $(E, \vdash)$ um sistema dedutivo. Dado $A \subseteq E$, o fecho dedutivo de $\mathrm{A}$ é o conjunto $\overline{\mathrm{A}}=\{\mathrm{x} \in \mathrm{E}: \mathrm{A} \vdash \mathrm{x}\}$. 
Definição 3.4: $\mathrm{Se}(E, \vdash)$ é um sistema dedutivo, então $A, B \subseteq E$ são dedutivamente equivalentes, o que é denotado por $A \sim B$, se $\bar{A}=\bar{B}$.

Proposição 3.5: $O$ sistema dedutivo $(E, \vdash)$ determina uma relação de ordem parcial $\leq$ sobre $E$, de tal modo que: $x \leq y \Leftrightarrow\{x\} \vdash y$.

Demonstração: A reflexividade de $\leq$ segue diretamente de (R1). Para verificarmos a transitividade de $\leq$ observemos que, se $x \leq y$ e $y \leq z$, então $\{x\} \vdash y$ e $\{y\} \vdash z$. De $\{y\} \vdash z$, pela $\left(R_{2}\right)$, temos $\{x\} \cup\{y\} \vdash z$. Daí $e\{x\} \vdash y$, pela $\left(R_{3}\right)$, temos que $\{x\} \vdash z$, isto é, $x \leq z$. Resta-nos mostrar a antissimetria que neste caso tem a seguinte configuração: se $x \leq y$ e $y \leq x$, então $\{x\} \vdash$ $y$ e $\{y\} \vdash x$ e isto nos diz que os conjuntos $\{x\}$ e $\{y\}$ são dedutivamente equivalentes, que é um tipo de "igualdade fraca" entre $x$ e $y$, ou uma igualdade lógica, na medida em que em uma dedução x e y são indistinguíveis.

Para caracterizar uma noção de lógica, na década de 1930, Alfred Tarski introduziu os conceitos de operador de consequência e de espaço de Tarski, os quais apresentamos a seguir junto com alguns resultados cujas demonstrações podem, em geral, serem encontradas em Martin e Pollard (1996).

Definição 3.6: Um operador de consequência sobre E é uma relação equipotente definida por uma função $\mathcal{C}: \mathcal{P}(E) \rightarrow \mathcal{P}(E)$ tal que, para todos $A, B \subseteq E$, são válidos:

(C1) $\mathrm{A} \subseteq \mathcal{C}(\mathrm{A})$ (autodedutibilidade)

( 2 2) $\mathrm{A} \subseteq \mathrm{B} \Rightarrow \mathcal{C}(\mathrm{A}) \subseteq \mathcal{C}(\mathrm{B})$ (monotonicidade)

(C3) $\mathcal{C}(\mathcal{C}(\mathrm{A})) \subseteq \mathcal{C}(\mathrm{A})$ (idempotência).

Para todo operador de consequência $\mathcal{C}$, segue de $\left(C_{1}\right)$ e $\left(C_{3}\right)$, que vale a igualdade $\mathcal{C}(\mathcal{C}(\mathrm{A}))=\mathcal{C}(\mathrm{A})$.

Definição 3.7: Um operador de consequência $\mathcal{C}_{\text {sobre }}$ é finitário quando, para todo $A \subseteq E$ vale:

$\left(C_{4}\right) \mathcal{C}(A)=\cup\left\{\mathcal{C}\left(A_{f}\right): A_{f}\right.$ é subconjunto finito de $\left.A\right\}$. 
Definição 3.8: Espaço de Tarski (sistema dedutivo, espaço de fecho, lógica) é qualquer par $(E, \mathcal{C})$, em que $E$ é um conjunto e $\mathcal{C}$ é um operador de consequência sobre $E$.

Definição 3.9: Seja $\mathcal{C}$ um operador de consequência sobre E. O conjunto $A \in \mathcal{P}(E)$ é fechado em $(E, \mathcal{C})$ quando $\mathcal{C}(\mathrm{A})=\mathrm{A}$, e A é aberto em $(E, \mathcal{C})$ quando seu complementar, denotado por $A^{C}$, é fechado em $\left(E, \mathcal{C}^{\prime}\right)$.

Proposição 3.10: $\mathrm{Se}(E, \mathcal{C})$ é um espaço de Tarski, então o domínio $E$ é fechado, o conjunto $\varnothing$ é aberto e, para todo $\mathrm{A} \subseteq \mathrm{E}$, o conjunto $\mathcal{C}(\mathrm{A})$ é fechado.

Proposição 3.11: Toda intersecção de conjuntos fechados em um espaço de Tarski $(E, \mathcal{C})$ é ainda um conjunto fechado. Toda união de conjuntos abertos é um aberto em ( $E, \mathcal{C})$.

Os conjuntos $\mathcal{C}(\varnothing)$ e $E$ correspondem ao menor e ao maior fechados, respectivamente, associados ao operador de consequência $\mathcal{C}$ sobre o conjunto $E$.

Definição 3.12: Um espaço de Tarski $(E, \mathcal{C})$ é vácuo se $\mathcal{C}(\varnothing)=\varnothing$.

Espaços topológicos determinam espaços de Tarski que são exemplos de espaços vácuos. Basta escolher $\mathcal{C}(A)=$ fecho topológico de $A$, isto é, a intersecção de todos os fechados que contêm $A$.

Proposição 3.13: Em um espaço de Tarski $(\mathrm{E}, \mathcal{C}$ ) vale a seguinte igualdade $\mathcal{C}(\mathrm{A} \cup \mathrm{B})=\mathcal{C}(\mathcal{C}(\mathrm{A}) \cup \mathrm{B})$.

Demonstração: Como $A \subseteq \mathcal{C}(A)$, então $\mathcal{C}(A \cup B) \subseteq \mathcal{C}(\mathcal{C}(A) \cup B)$. Por outro lado, $\mathcal{C}(A) \subseteq \mathcal{C}(A \cup B)$ e $B \subseteq \mathcal{C}(A \cup B)$ e, então $\mathcal{C}(A) \cup B \subseteq \mathcal{C}(A \cup B)$ e, portanto, $\mathcal{C}(\mathcal{C}(A) \cup B) \subseteq \mathcal{C}(A \cup B)$.

Observação: $\mathrm{Se}(E, \vdash)$ é um sistema dedutivo e $A \subseteq E$, então o fecho dedutivo de $\mathrm{A}$, pode ser denotado por $\overline{\mathrm{A}}$ ou $\mathcal{C}(\mathrm{A})=\{\mathrm{x} \in \mathrm{E}: \mathrm{A} \vdash \mathrm{x}\}$. 
A demonstração dos seguintes dois resultados, pode ser encontrada em Feitosa, Moreira e Soares (2016).

Proposição 3.14: Se $(E, \vdash)$ é um sistema dedutivo, então o fecho dedutivo determina um operador de consequência de Tarski.

A recíproca deste resultado também é bem simples.

Proposição 3.15: $\mathrm{Se}(E, \mathcal{C})$ é um espaço de Tarski, então a relação não equipotente $\vdash$ definida a partir de $\mathcal{C}$ por $A \vdash x \Leftrightarrow x \in \mathcal{C}(A)$ é uma relação de consequência.

Desta forma, temos uma equivalência extencional entre os conceitos de relação de consequência e operador de Tarski.

\section{4 Álgebra implicacional}

Após todas as considerações pregressas, estamos prontos para introduzir a estrutura algébrica que nos interessa. Na próxima seção discorreremos sobre a lógica que estará associada a essa álgebra e, finalmente, estabeleceremos, de uma maneira muito técnica, específica e usual, a adequação entre tais sistemas formais.

O conceito de álgebra implicacional, para o qual usaremos a notação iálgebra, procura formalizar uma noção lógica básica chamada implicação, ou condicional, no âmbito de uma estrutura algébrica. No trabalho que ora apresentamos, esquecemos que existem outros operadores lógicos importantes e concentramo-nos na implicação.

A abordagem deste tópico é feita de modo similar a Rasiowa (1974), que denominou esta estrutura de álgebra implicativa positiva.

Por tratarmos de uma noção de condicional, esta formalização resgata aspectos do "se, ... então", que nos conduzem à uma noção de ordem. 
Definição 4.1: Uma álgebra implicacional, ou iálgebra, é uma terna $A$ $=(A, 1, \leadsto)$ em que $A$ é um conjunto, 1 é uma constante tal que $1 \in A$, e $u$ é uma operação binária sobre $A$, que satisfaz as seguintes condições, para todos $a, b, c \in \mathrm{A}$ :

$$
\begin{aligned}
& \text { (a) } a \rightsquigarrow 1=1 \\
& \left(a_{2}\right) \text { se } a \rightsquigarrow b=1 \text { e } b \rightsquigarrow a=1 \text {, então } a=b \\
& \left(a_{3}\right) a \rightsquigarrow(b \rightsquigarrow a)=1 \\
& \left(a_{4}\right)(a \rightsquigarrow(b \rightsquigarrow c)) \cdots((a \rightsquigarrow b) \rightsquigarrow(a \rightsquigarrow c))=1 .
\end{aligned}
$$

Em uma abordagem mais precisa, porém não usual, enxergando A como um sistema formal, diríamos ter um alfabeto formado: pelos elementos de A; pelo seu elemento distinguido 1; pela operação binária u; pela relação de identidade =; e pelos símbolos ) e (. A partir destes obteríamos as fórmulas e assim por diante.

É nossa intenção que a operação $\leadsto$ induza uma ordem sobre $A$. Assim, a condição $\left(a_{1}\right)$ destaca que o elemento 1 é um máximo em $A$. A condição $\left(\mathrm{a}_{2}\right)$ introduz a antissimetria em $A$. Veremos que as condições $\left(\mathrm{a}_{3}\right)$ e $\left(\mathrm{a}_{4}\right)$ dão conta de gerar a reflexividade e a transitividade em $A$.

Proposição 4.2: Se $a \rightsquigarrow b=1$ e $a=1$, então $b=1$.

Demonstração: Se $a \rightsquigarrow b=1$ e $a=1$, então $1 \leadsto b=1$. Como por $\left(a_{1}\right), b \rightsquigarrow 1$ $=1$, então por $\left(a_{2}\right)$, segue que $a=b=1$.

Proposição 4.3: Se $A=(\mathrm{A}, 1, \rightsquigarrow)$ é uma iálgebra e $a, b, c \in \mathrm{A}$, então:

(i) $a \rightsquigarrow a=1$

(ii) se $a \rightsquigarrow b=1$ e $b \leadsto c=1$, então $a \rightsquigarrow c=1$

Demonstração: (i)

1. $(a \rightsquigarrow((a \rightsquigarrow a) \cdots a)) \cdots((a \rightsquigarrow(a \rightsquigarrow a)) \cdots(a \rightsquigarrow a))=1$

2. $(a \rightsquigarrow((a \rightsquigarrow a) \cdots a))=1$

3. $1 \rightsquigarrow((a \rightsquigarrow(a \rightsquigarrow a)) \rightsquigarrow(a \rightsquigarrow a))=1 \quad$ Substituição de (2) em (1)

4. $((a \rightsquigarrow(a \rightsquigarrow a)) \rightsquigarrow(a \rightsquigarrow a))=1$ Proposição 4.2 em (3)

5. $(a \rightsquigarrow(a \rightsquigarrow a))=1$ 

6. $(1 m(a m a))=1$
Substituição de (5) em (4)
7. $a \leadsto a=1$
Proposição 4.2 em (6)
(ii)
1. $a \leadsto b=1$
premissa
2. $b \rightsquigarrow c=1$
premissa
3. $a m 1=1$
4. $a m(b m c)=1$
Substituição de (2) em (3)
5. $(a \cdots(b \cdots c)) \cdots((a m b) \cdots(a m c))=1$
6. $1 \cdots((a \rightsquigarrow b) \cdots(a \rightsquigarrow c))=1$
(4 e a a $_{4}$
7. $((a m b) \rightsquigarrow(a \rightsquigarrow c))=1$
Proposição 4.2 em (6)
8. $(1 \cdots(a \rightsquigarrow c))=1$
Substituição de (1) em (7)
9. $a m c=1$ Proposição 4.2 em (8).

Definição 4.4: Se $A=(A, 1, \rightsquigarrow)$ é uma iálgebra, então definimos a relação $\leq$ por:

$$
a \leq b \Leftrightarrow a \leadsto b=1 .
$$

Proposição 4.5: A relação $\leq$ é uma ordem parcia1 e o elemento 1 é máximo em $A=(A, 1, \rightsquigarrow)$ para a ordem $\leq$.

Demonstração: Segue de $\left(a_{1}\right),\left(a_{2}\right)$ e Proposição 4.3.

Considerando a definição da ordem $\leq$, acima, temos os seguintes resultados.

Proposição 4.6: Se $A=(\mathrm{A}, 1, \cdots)$ é uma iálgebra e $a, b, c \in \mathrm{A}$, então:

(i) $a \leq b \leadsto a$

(ii) $a \cdots(b \cdots c) \leq(a \rightsquigarrow b) \cdots(a \rightsquigarrow c)$

(iii) $b \leq c \Rightarrow a \rightsquigarrow b \leq a \rightsquigarrow c$

(iv) $a \leq b \leadsto c \Leftrightarrow b \leq a \cdots c$

Demonstração: Os itens (i) e (ii) decorrem de $\left(\mathrm{a}_{3}\right)$ e $\left(\mathrm{a}_{4}\right)$. 
(iii) Como $b \leq c$, então $b \leadsto c=1$. Assim, segue de $\left(a_{1}\right)$ que $a \rightsquigarrow(b \leadsto c)=$ 1. Por $\left(a_{4}\right)$ temos $(a \cdots(b \leadsto c)) \cdots((a \cdots b) \cdots(a \cdots c))=1$, logo pela Proposição 4.2 segue que $(a m b) \leadsto(a \cdots c)=1$, isto é, $a m b \leq a \rightsquigarrow c$.

(iv) Se $a \leq b \leadsto c$, então $a \rightsquigarrow(b \leadsto c)=1$. Da Proposição 4.2 e (a a $_{4}$, segue que $(a \rightsquigarrow b) \cdots(a \rightsquigarrow c)=1$, ou seja, $(a \rightsquigarrow b) \leq(a \rightsquigarrow c)$. Assim, utilizando (iii) teremos que $b \leadsto(a \rightsquigarrow b) \leq b \backsim(a \cdots c)$. Agora, por $\left(a_{3}\right),\left(a_{1}\right)$ e Proposição 4.2 seguirá que $b \leadsto(a \rightsquigarrow c)=1$. A recíproca é análoga.

Se em uma iálgebra $A=(A, 1, \leadsto)$ fixarmos um elemento arbitrário $c$ de $\mathrm{A}$ e definirmos as funções $\mathrm{f}, \mathrm{g}: A \rightarrow A$ tal que $\mathrm{f}(a)=a \leadsto c$ e $\mathrm{g}(b)=b \leadsto c$, quaisquer que sejam $a, b \in \mathrm{A}$, decorrerá da Proposição 4.6 (iv) que o par $(f, g)$ determina uma conexão de Galois, conforme Definição 2.14. Esse fato produzirá alguns resultados que são descritos na próxima proposição.

Proposição 4.7: Se $A=(\mathrm{A}, 1, \rightsquigarrow)$ é uma iálgebra e $a, b, c \in \mathrm{A}$, então:

(i) $a \leq(a \leadsto c) \leadsto c$

(ii) $a \leq b \Rightarrow b \leadsto c \leq a \rightsquigarrow c$

(iii) $a \rightsquigarrow c=((a \rightsquigarrow c) \leadsto c) \cdots c$

(iv) $1 m c=c$

(v) $a \cdots(b \leadsto c)=b \leadsto(a \rightsquigarrow c)$

(vi) $(a \rightsquigarrow b) \cdots(a \cdots c) \leq a \rightsquigarrow(b \leadsto c)$

(vii) $(a \cdots b) \rightsquigarrow(a \cdots c)=a \cdots(b \leadsto c)$

(viii) $a \rightsquigarrow(a \rightsquigarrow b)=a \rightsquigarrow b$.

Demonstração: Fixamos $c \in A$ e definindo as funções $f$ e $g$ conforme descrito acima teremos a conexão de Galois $(f, g)$. Decorrem:

(i) Pela Proposição 2.15 (iii), como $a \leq g(f(a))$ então, $a \leq g(a \rightsquigarrow c)$ e $a \leq(a \rightsquigarrow c) \rightsquigarrow c$.

(ii) Pela Proposição 2.15 (ii), se $a \leq b$, então $g(b) \leq g(a)$, isto é, $b \leadsto c \leq a \rightsquigarrow c$.

(iii) Pela Proposição 2.16, como $f(a)=f(g(f(a)))$, segue que $a m c=$ $f((a \rightsquigarrow c) \rightsquigarrow c)=((a \rightsquigarrow c) \rightsquigarrow c) \rightsquigarrow c$. 
(iv) Por (i) desta proposição temos que $1 \leq(1 \backsim c) \leadsto c$ e, então, por $\left(a_{1}\right),(1 \rightsquigarrow c) \leadsto c=1$. Assim, $1 \leadsto c \leq c$. Da Proposição 4.6 (i), temos que $c \leq$ $1 \leadsto c$. Portanto, $1 \leadsto c=c$.

(v) Pela Proposição 4.6 (ii), $a \rightsquigarrow(b \leadsto c) \leq(a \rightsquigarrow b) \leadsto(a \rightsquigarrow c)$. Pela mesma Proposição 4.6 (iv), temos $(a \rightsquigarrow b) \leq(a \rightsquigarrow(b \leadsto c)) \rightsquigarrow(a \rightsquigarrow c)$. Como $b$ $\leq a \rightsquigarrow b$ então, segue que $b \leq(a \rightsquigarrow(b \rightsquigarrow c)) \rightsquigarrow(a \rightsquigarrow c)$. Mais uma vez pela Proposição 4.6 (iv), temos $a \rightsquigarrow(b \leadsto c) \leq b \leadsto(a \cdots c)$. Trocando $a$ por $b$, segue $b \leadsto(a \cdots c) \leq a \cdots(b \cdots c)$ e, portanto, vale a igualdade.

(vi) Como, por 4.6 (i), $b \leq a \rightsquigarrow b$, então, pela Proposição 4.7 (ii), temos que $(a \rightsquigarrow b) \cdots(a \rightsquigarrow c) \leq b \rightsquigarrow(a \rightsquigarrow c)=a \rightsquigarrow(b \leadsto c)$, pela Proposição 4.7 (v).

(vii) Segue da Proposição 4.6 (ii) e da Proposição 4.7 (vi).

(viii) Pela Proposição 4.6 (i), $a \backsim b \leq a \backsim(a \backsim b)$. Por outro lado, pela Proposição 4.6 (ii), $a \rightsquigarrow(a \rightsquigarrow b) \leq(a \rightsquigarrow a) \cdots(a \rightsquigarrow b)=1 \cdots(a \rightsquigarrow b)=a \rightsquigarrow b$. Logo, vale a igualdade.

Um conceito importante, do qual faremos uso oportunamente, é apresentado a seguir e chamado de Filtro Implicativo. Assim, como convencionamos chamar a álgebra implicacional de iálgebra, adotaremos a expressão ifiltro para os filtros implicativos.

Definição 4.8: Seja $A=(A, 1, m)$ uma iálgebra. Um filtro implicativo, ou ifiltro, em $A$ é um subconjunto não vazio $\mathbf{F} \subseteq \mathrm{A}$ tal que, para todos $a, b \in \mathrm{A}$ : se $a \in \mathbf{F}$ e $a \leq b$, então $b \in \mathbf{F}$.

Certamente, o elemento 1 de A pertence a todo ifiltro $\mathbf{F}$.

Proposição 4.9: Se $A=(A, 1, \rightsquigarrow)$ é uma iálgebra e $a \in A$, então:

(i) o conjunto $a \rightarrow=\{b \in \mathrm{A}: a \leq b\}$ é um ifiltro;

(ii) se $\mathbf{F} \subseteq$ A é um ifiltro, então $a \in \mathbf{F} \Leftrightarrow a \rightarrow \subseteq \mathbf{F}$. 
Demonstração: (i) Se $b \in a \rightarrow$ e $b \leq c$, pela lei de formação de $a \rightarrow$, $a$ $\leq b$ e, desta forma, $a \leq c$. Logo, $c \in a \rightarrow$. Deste modo, $a \rightarrow=\{b \in \mathrm{A}: a \leq$ b\} é um ifiltro.

(ii) $(\Rightarrow)$ Se $a \in \mathbf{F}$ e $a \leq b$, pela definição de ifiltro, $b \in \mathbf{F}$. Logo, $\mathbf{F}$ tem como elementos todos os elementos de $a \rightarrow$, ou seja, $a \rightarrow \subseteq \mathbf{F}$.

$(\Leftarrow)$ Se $a \rightarrow \subseteq \mathbf{F}$, como $a \in a \rightarrow$, então $a \in \mathbf{F}$.

Definição 4.10: O filtro $a \rightarrow$ é denominado filtro implicativo gerado por a, ou simplesmente ifiltro gerado por a.

Proposição 4.11: $\operatorname{Se} A=(\mathrm{A}, 1, \rightsquigarrow)$ é uma iálgebra e $\mathbf{F} \subseteq \mathrm{A}$ é um ifiltro sobre $A$, então:

(i) o conjunto $\{1\} \subseteq$ A é um ifiltro de $A$;

(ii) o conjunto $\{1\} \subseteq$ A está contido em todo ifiltro de $A$.

Demonstração: (i) $\{1\}=1 \rightarrow$.

(ii) Seja $\mathbf{F}$ um ifiltro qualquer de $A$. Como $1 \in A$ e para todo $a \in \mathbf{F}$, segue que $a \leq 1$, então, pela Definição 4.8 , segue que $1 \in \mathbf{F}$. Logo, $\{1\} \subseteq$ $\mathrm{F}$, para todo $\mathrm{F}$ de $A$.

Proposição 4.12: Sejam $A=(A, 1, \rightsquigarrow)$ uma iálgebra e $S \subseteq A$. A intersecção de todos os ifiltros de $A$ que contêm $S$ é um ifiltro.

Demonstração: Seja $[\mathrm{S}]=\cap\{\mathrm{F} \lambda \subseteq \mathrm{A}: \mathbf{F} \boldsymbol{\lambda}$ é ifiltro de $A$ e $\mathrm{S} \subseteq \mathbf{F} \lambda\}$. Precisamos verificar que [S] é um ifiltro de $A$.

Sejam $a, b \in \mathrm{A}$. Se $a \in[\mathrm{S}]$ e $a \leq b$, então para todo $\lambda, a \in \mathbf{F} \lambda$. Como cada $\mathbf{F} \lambda$ é ifiltro, então $b \in \mathbf{F} \lambda$ e, portanto, $b \in[\mathrm{S}]$.

Logo, $[\mathrm{S}]=\cap\{\mathbf{F} \lambda \subseteq \mathrm{A}: \mathbf{F} \lambda$ é ifiltro e $\mathrm{S} \subseteq \mathbf{F} \lambda\}$ é um ifiltro de $A$. 
Definição 4.13: O ifiltro [S] é denominado filtro gerado por S, ou simplesmente ifiltro gerado por $S$.

Definição 4.14: Sejam $A=(\mathrm{A}, 1, \cdots)$ uma iálgebra e $\mathbf{F} \subseteq \mathrm{A}$ um ifiltro de $A$. O ifiltro $\mathbf{F}$ é próprio se $\mathbf{F} \neq \mathrm{A}$.

Definição 4.15: Sejam $A=(A, 1, \rightsquigarrow)$ uma iálgebra e $\mathbf{F} \subseteq \mathrm{A}$ um ifiltro. O ifiltro $\mathbf{F}$ é maximal se ele é próprio e:

(i) para todo ifiltro $\mathrm{F}^{*}$ de $A$, se $\mathrm{F} \subseteq \mathrm{F}^{*}$, então $\mathrm{F}^{*}=\mathrm{F}$ ou $\mathrm{F}^{*}=\mathrm{A}$.

Assim, um ifiltro maximal F não está contido em qualquer ifiltro maximal distinto dele.

Definição 4.16: Sejam $A=(A, 1, \rightsquigarrow)$ uma iálgebra e $\mathbf{F} \subseteq \mathrm{A}$ um ifiltro. $\mathrm{O}$ ifiltro $\mathbf{F}$ é dito irredutível se ele é próprio e, para todos dois ifiltros $\mathbf{F}_{\mathbf{1}}$, $\mathbf{F}_{2}$, se $\mathbf{F}==\mathbf{F}_{1} \cap \mathbf{F}_{2}$, então $\mathbf{F}=\mathbf{F}_{1}$ ou $\mathbf{F}=\mathbf{F}_{2}$.

Proposição 4.17: Se $A=(\mathrm{A}, 1, \leadsto)$ é uma iálgebra, então as seguintes condições são equivalentes para todo filtro $\mathbf{F} \subseteq \mathrm{A}$ :

(i) F é ifiltro maximal

(ii) $\mathbf{F}$ é ifiltro irredutível.

Demonstração: Ifiltros maximais e irredutíveis têm que ser próprios. Se $\mathbf{F}$ é maximal e $\mathbf{F}=\mathbf{F}_{\mathbf{1}} \cap \mathbf{F}_{\mathbf{2}}$, com $\mathbf{F}_{\mathbf{1}}$ e $\mathbf{F}_{\mathbf{2}}$ ifiltros, então $\mathbf{F}=\mathbf{F}_{\mathbf{1}}$ ou $\mathbf{F}=\mathbf{F}_{\mathbf{2}}$ e, portanto, F é irredutível. Por outro lado, se F é irredutível, só pode ser isubfiltro dele mesmo e de A. Logo, F é maximal.

Lema 4.18: Sejam $A=(A, 1, \leadsto)$ uma iálgebra e $F$ uma cadeia de ifiltros em $A$. Então:

(i) UF é ifiltro de $A$;

(ii) se $\mathbf{x} \notin \mathbf{F}$, para todo elemento $\mathbf{F}$ de $F$, então $\mathbf{x} \notin \cup F$. 
Proposição 4.19: Sejam $A=(A, 1, \leadsto)$ uma iálgebra e $\mathbf{F}$ um ifiltro de $A$. Se $x \notin \mathbf{F}$, então existe um ifiltro irredutível $\mathbf{F}^{*}$ tal que $\mathbf{F} \subseteq \mathrm{F}^{*}$ e $x \notin \mathbf{F}^{*}$.

Demonstração: Seja F o conjunto ordenado pela inclusão de conjuntos $\subseteq$ de todos os ifiltros sobre $A$ que contêm $\mathbf{F}$ e não contêm $\mathrm{x}$. Pelo lema anterior, toda cadeia em $F$ tem um limitante superior. Então, pelo Lema de Zorn (Proposição 2.10), existe um elemento maximal F* em F. Pela definição de $F, \quad \mathbf{F} \subseteq \mathbf{F}^{*}$ e $\mathbf{x} \notin \mathbf{F}^{*}$.

Resta verificarmos que $\mathbf{F}^{*}$ é irredutível. Se $\mathbf{F}^{*}=\mathbf{F}_{\mathbf{1}} \cap \mathbf{F}_{\mathbf{2}}$ e $\mathbf{F}_{\mathbf{1}}$ e $\mathbf{F}_{\mathbf{2}}$ são ifiltros, então $\mathbf{F}^{*} \subseteq \mathbf{F}_{\mathbf{1}}, \mathbf{F}^{*} \subseteq \mathbf{F}_{\mathbf{2}}$ e $\mathbf{x} \notin \mathbf{F}_{\mathbf{1}}$ ou $\mathbf{x} \notin \mathbf{F}_{\mathbf{2}}$. Deste modo, ou $\mathbf{F}_{\mathbf{1}} \in F$ ou $\mathbf{F}_{2} \in F$. Como $\mathbf{F}^{*}$ é maximal em $F$ e $\mathbf{F}^{*} \subseteq \mathbf{F}_{1}, \mathbf{F}^{*} \subseteq \mathbf{F}_{2}$, então $\mathbf{F}^{*}=\mathbf{F}_{1}$ ou $\mathbf{F}^{*}=\mathbf{F}_{2}$.

A seguir, veremos a formalização dessa noção de implicação no ambiente de uma lógica proposicional.

\section{Lógica implicacional}

Nesta seção apresentamos a lógica implicacional que denotaremos por iL. Trata-se de uma lógica proposicional com um único operador lógico para representar a implicação.

Por portar um único operador, podemos destacar muitos aspectos lógicos da implicação. Veremos como este operador, com as propriedades aqui indicadas, conduz naturalmente ao conceito de ordem.

A linguagem $L$ de iL é construída a partir de um símbolo $\rightarrow$, para a implicação, das variáveis proposicionais, cujo conjunto denotaremos $\operatorname{Var}(L)=\left\{p_{1}, p_{2}, p_{3}, \ldots\right\}$, além dos símbolos) e (.

Definição 5.1: $O$ conjunto $\operatorname{Var}(L)=\left\{p_{1}, p_{2}, p_{3}, \ldots\right\}$, das variáveis proposicionais de $\mathrm{L}$, corresponde às fórmulas atômicas da linguagem $\mathrm{L}$. 
Definição 5.2: O conjunto das fórmulas de $L$, denotado por For $(L)$, é definido indutivamente por:

(i) cada variável $p_{\mathrm{i}}$ está em For $(L)$;

(ii) se $\varphi, \psi \in$ For(L), então $\varphi \rightarrow \psi \in$ For $(L)$;

(iii) nada mais pertence a $\operatorname{For}(\mathrm{L})$.

O sistema axiomático dedutivo de iL fica determinado pelos seguintes itens:

Esquemas de Axiomas:

$(\mathrm{A} 1) \varphi \rightarrow(\psi \rightarrow \varphi)$

(A2) $(\varphi \rightarrow(\psi \rightarrow \sigma)) \rightarrow((\varphi \rightarrow \psi) \rightarrow(\varphi \rightarrow \sigma))$.

Regra de Dedução:

(MP) $\varphi, \varphi \rightarrow \psi \vdash \psi$.

Com as definições de dedução e demonstração da Seção 1, podemos dar exemplos de resultados válidos em iL.

Proposição 5.3: (i) $\vdash \varphi \rightarrow \varphi$

(ii) $\varphi \rightarrow \psi, \psi \rightarrow \sigma \vdash \varphi \rightarrow \sigma$

(iii) $\varphi \rightarrow(\psi \rightarrow \sigma) \vdash \psi \rightarrow(\varphi \rightarrow \sigma)$.

Demonstração: (i)

1. $(\varphi \rightarrow((\varphi \rightarrow \varphi) \rightarrow \varphi)) \rightarrow((\varphi \rightarrow(\varphi \rightarrow \varphi)) \rightarrow(\varphi \rightarrow \varphi))$ instância de (A2)

2. $\varphi \rightarrow((\varphi \rightarrow \varphi) \rightarrow \varphi) \quad$ instância de (A1)

3. $(\varphi \rightarrow(\varphi \rightarrow \varphi)) \rightarrow(\varphi \rightarrow \varphi) \quad$ MP em 1 e 2

4. $\varphi \rightarrow(\varphi \rightarrow \varphi) \quad$ instância de (A1)

5. $\varphi \rightarrow \varphi \quad$ MP em 3 e 4.

(ii)

1. $\varphi \rightarrow \psi$

p. 
2. $\psi \rightarrow \sigma$

p.

3. $(\psi \rightarrow \sigma) \rightarrow(\varphi \rightarrow(\psi \rightarrow \sigma))$

4. $\varphi \rightarrow(\psi \rightarrow \sigma)$ MP em 2 e 3

5. $(\varphi \rightarrow(\psi \rightarrow \sigma)) \rightarrow((\varphi \rightarrow \psi) \rightarrow(\varphi \rightarrow \sigma))$

6. $(\varphi \rightarrow \psi) \rightarrow(\varphi \rightarrow \sigma)$ MP em 4 e 5

7. $\varphi \rightarrow \sigma$ MP em 1 e 6.

(iii)

1. $\varphi \rightarrow(\psi \rightarrow \sigma)$

p.

2. $(\varphi \rightarrow(\psi \rightarrow \sigma)) \rightarrow((\varphi \rightarrow \psi) \rightarrow(\varphi \rightarrow \sigma))$

3. $(\varphi \rightarrow \psi) \rightarrow(\varphi \rightarrow \sigma)$ MP em 1 e 2

4. $\psi \rightarrow(\varphi \rightarrow \psi)$ $\mathrm{Ax}_{1}$

5. $\psi \rightarrow(\varphi \rightarrow \sigma)$ (ii) em 3 e 4 .

Teorema 5.4: (Teorema da Dedução) Seja $\Delta \cup\{\varphi, \psi\} \subseteq$ For(L). Se $\Delta \cup\{\varphi\}$ $\vdash \psi$, então $\Delta \vdash \varphi \rightarrow \psi$.

Demonstração: A mesma de Feitosa e Paulovich (2005).

Proposição 5.5: Seja $\Delta \cup\{\varphi, \psi\} \subseteq$ For(L). Se $\Delta \vdash \varphi \rightarrow \psi$, então $\Delta \cup\{\varphi\} \vdash \psi$. Demonstração:
1. $\Delta \cup\{\varphi\} \vdash \varphi \rightarrow \psi$
$\Delta \subseteq \Delta \cup\{\varphi\}$ e p.
2. $\Delta \cup\{\varphi\} \vdash \varphi$
$\varphi \in \Delta \cup\{\varphi\}$
3. $\Delta \cup\{\varphi\} \vdash \psi$ MP em 1 e 2

Corolário 5.6: $\operatorname{Se} \varphi, \psi, \sigma \in \operatorname{For}(L)$ :
(i) $\varphi \rightarrow(\psi \rightarrow \sigma), \varphi \rightarrow \psi \vdash \varphi \rightarrow \sigma$.

Demonstração: (i)
1. $\varphi \rightarrow(\psi \rightarrow \sigma)$
p.
2. $\psi$
p. 
3. $\varphi$

4. $\psi \rightarrow \sigma$

5. $\sigma$ pp.

MP em 1 e 3

MP em 2 e 4

Logo, $\varphi \rightarrow(\psi \rightarrow \sigma), \varphi \rightarrow \psi, \varphi \vdash \sigma$ e, pelo TD, $\varphi \rightarrow(\psi \rightarrow \sigma), \varphi \rightarrow \psi \vdash \varphi \rightarrow \sigma$.

\section{Adequação}

Agora, apresentamos a adequação entre a lógica iL e as iálgebras.

Denotaremos uma iálgebra genérica $A=(A, 1, \leadsto)$ simplesmente por $A$.

Definição 6.1: Uma valoração restrita é uma função u^: $\operatorname{Var}(\mathrm{L}) \rightarrow A$, que interpreta cada variável de $L$ em um elemento de $A$.

Definição 6.2: Para $p, \varphi, \psi \in$ For $(L)$, uma valoração ou interpretação é uma função u: For $(L) \rightarrow A$ que estende de modo natural e único a valoração restrita do seguinte modo:

(i) $u(p)=u \wedge(p)$

(ii) $U(\varphi \rightarrow \psi)=U(\varphi) \leadsto U(\psi)$.

Os símbolos de operadores do lado esquerdo representam operadores lógicos, enquanto os símbolos de operadores do lado direito representam os operadores algébricos de $A$.

Definição 6.3: Uma valoração U: For $(\mathrm{L}) \rightarrow A$ é um modelo para um conjunto $\Gamma \subseteq \operatorname{For}(L)$ se $u(\varphi)=1$, para toda fórmula $\varphi \in \Gamma$.

Também dizemos que o par $(U, A)$ satisfaz o conjunto $\Gamma$ e indicamos este modelo de $\Gamma$ por $(U, A) \vDash \Gamma$. Se o conjunto $\Gamma$ tem uma única fórmula $\gamma$, denotamos a satisfação por $(U, A) \vDash \gamma$. 
Definição 6.4:Se $A$ é uma iálgebra e $\varphi \in$ For( $(\mathrm{L})$, então a fórmula $\varphi$ é válida em $A$ se toda valoração u: For $(\mathrm{L}) \rightarrow A$ é um modelo para $\varphi$.

Neste caso, indicamos que $A$ é um modelo para $\varphi$ por $A \vDash \varphi$ e que $A$ é um modelo para $\Gamma$ por $A \models \Gamma$.

Definição 6.5: Uma fórmula p é válida quando ela é válida em toda iálgebra.

Denotamos que $\varphi$ é válida por $\vDash \varphi$ e que todo modelo de $\Gamma$ é também modelo de $\varphi$ por $\Gamma \vDash \varphi$

Teorema 6.6: (Correção) Sejam $\Gamma \subseteq$ For( $(L)$ em uma iL e $A$ uma iálgebra qualquer. Se $\Gamma \vdash \gamma$, então $\Gamma \vDash \gamma$.

Demonstração: Por indução sobre o comprimento da dedução $\Gamma \vdash \gamma$.

Seja $(U, A)$ um modelo qualquer para $\Gamma$. Se a dedução tem comprimento 1, então $\gamma \in \Gamma$ ou $\gamma$ é um axioma de iL.

Se $\gamma \in \Gamma$ e $A \vDash \Gamma$, então $A \vDash \gamma$.

Se $\gamma$ é um axioma, então $\gamma$ é $\varphi \rightarrow(\psi \rightarrow \varphi)$ ou $\gamma$ é $(\varphi \rightarrow(\psi \rightarrow \sigma)) \rightarrow$ $((\varphi \rightarrow \psi) \rightarrow(\varphi \rightarrow \sigma))$. Da definição da iálgebra, temos que para toda valoração $\mathrm{u}$ em qualquer iálgebra $A, \mathrm{u}(\varphi \rightarrow(\psi \rightarrow \varphi))=1$ e $\mathrm{u}((\varphi \rightarrow(\psi \rightarrow \sigma)) \rightarrow((-$ $\varphi \rightarrow \psi) \rightarrow(\varphi \rightarrow \sigma)))=1$.

Agora, consideremos que o último passo da dedução $\Gamma \vdash \gamma$ seja por uma aplicação da regra MP: $\Gamma \vdash \sigma$ e $\Gamma \vdash \sigma \rightarrow \gamma / \Gamma \vdash \gamma$.

Por hipótese de indução, temos que $\mathrm{U}(\sigma)=1$ e $\mathrm{U}(\sigma \rightarrow \gamma)=1$. Da iálgebra temos que $\mathrm{U}(\sigma) \leq \mathrm{U}(\gamma)$. Logo, $\mathrm{U}(\gamma)=1 \mathrm{e}$, portanto, a regra única MP preserva a validade de iL relativa aos modelos $(U, A)$.

Para completarmos a adequação devemos mostrar a recíproca deste teorema. Nesta demonstração surgirá o conceito de álgebra de Lindenbaum de iL. 
Definição 6.7: Chamamos de álgebra das fórmulas de iL a estrutura algébrica dada pelo par (For $(\mathrm{L}), \rightarrow$ ).

Definição 6.8: Para $\varphi, \psi \in \operatorname{For}(L)$, definimos a relação $\equiv$ por:

$$
\varphi \equiv \psi \Leftrightarrow \vdash \varphi \rightarrow \psi \text { e } \vdash \psi \rightarrow \varphi .
$$

Proposição 6.9: A relação 三 é uma congruência.

Demonstração: É uma congruência por ser uma relação de equivalência que preserva o único operador envolvido $\rightarrow$. Verificamos que $\equiv$ é uma relação de equivalência.

A relação é reflexiva: para toda fórmula $\varphi \in \operatorname{For}(L), \vdash \varphi \rightarrow \varphi$ e então $\varphi \equiv \varphi$.

A relação é simétrica: se $\varphi \equiv \psi$, então $\vdash \varphi \rightarrow \psi$ e $\vdash \psi \rightarrow \varphi$. Logo, $\psi \equiv \varphi$.

A relação é transitiva: se $\varphi \equiv \psi$ e $\psi \equiv \sigma$, então $\vdash \varphi \rightarrow \psi, \vdash \psi \rightarrow \varphi, \vdash$ $\psi \rightarrow \sigma$ e $\vdash \sigma \rightarrow \psi$. Logo, $\vdash \varphi \rightarrow \sigma$ e $\vdash \sigma \rightarrow \varphi \Leftrightarrow \varphi \equiv \sigma$.

Assim, a relação $\equiv$ é uma equivalência que preserva $\rightarrow$, uma vez que, se $\varphi \equiv \psi$ e $\sigma \equiv \lambda$, então $\varphi \rightarrow \sigma \equiv \psi \rightarrow \lambda$.

Definição 6.10: A classe de equivalência de $\varphi$ módulo 三 é dada por:

$$
[\varphi]=\{\psi \in \operatorname{For}(L): \psi \equiv \varphi\} \text {. }
$$

Definição 6.11: A álgebra de Lindenbaum de iL, que será denotada por $A(L)$, é a álgebra quociente:

$$
A(L)=(\text { For }(L) \mid \equiv, \rightarrow \equiv, T) \text {, tal que: }
$$

(i) $[\varphi] \rightarrow \equiv[\psi]=[\varphi \rightarrow \psi]$

(ii) $1 \equiv=[\varphi \rightarrow \varphi]=\top$.

O elemento $T$ representa a classe de equivalência dos teoremas da iL. Não escreveremos mais o subíndice $\equiv$ da álgebra acima.

Proposição 6.12: A álgebra $A(L)$ é implicacional. 
Demonstração: Segue dos axiomas (A1), (A2) e 5.3 (i).

Proposição 6.13: Na álgebra $A(L)$ vale: $[\varphi] \leq[\psi] \Leftrightarrow \vdash \varphi \rightarrow \psi$. Demonstração: $[\varphi] \leq[\psi] \Leftrightarrow[\varphi] \rightarrow[\psi]=\top \Leftrightarrow \vdash \varphi \rightarrow \psi$.

Definição 6.14: Uma teoria $\Delta$ é um conjunto não vazio de fórmulas fechado para a dedução, isto é, $\varphi \in \Delta$ se, e somente se, $\Delta \vdash \varphi$.

Definição 6.15: Uma teoria $\Delta$ de iL é maximal se para qualquer teoria $\Delta^{*}$ de iL, se $\Delta \subseteq \Delta^{*}$, então $\Delta^{*}=\Delta$ ou $\Delta^{*}=\operatorname{For}(L)$.

Seja T o conjunto de todas as teorias maximais da iL.

Definição 6.16: Para $\varphi \in$ For $(L)$, seja $\|\varphi\|=\{\Delta \in \mathbf{T}: \varphi \in \Delta\}$.

Proposição 6.17: Para $\varphi, \psi \in$ For $(L),\|\varphi\| \subseteq\|\psi\|$ se, e somente se, $\vdash \varphi \rightarrow \psi$.

Demonstração: $(\Rightarrow)$ Se $\forall \varphi \rightarrow \psi$, então existe $\Delta \in \mathbf{T}$ tal que $\varphi \rightarrow \psi \notin \Delta$. Uma tal teoria deve ser tal que $\Delta \vdash \varphi$ e $\Delta H \psi$, isto é, $\|\varphi\| \nsubseteq\|\psi\|$.

$(\Leftarrow)$ Se $\Delta \in\|\varphi\|, \Delta \vdash \varphi$. Como $\vdash \varphi \rightarrow \psi$, então $\Delta \vdash \varphi \rightarrow \psi$ e, por MP, $\Delta$ $\vdash \psi$. Logo $\Delta \in\|\psi\|$.

Definição 6.18: Seja $A_{\|}=(\{\|\varphi\|: \varphi \in \operatorname{For}(L)\}, \rightarrow, 1)$, definida por:

(i) $1=\|\varphi \rightarrow \varphi\|=\mathbf{T}$

(ii) $\|\varphi\| \nrightarrow\|\psi\|=\|\varphi \rightarrow \psi\|$.

Proposição 6.19: Para $\varphi, \psi \in$ For $(\mathrm{L}), \vdash \varphi \rightarrow \psi$ se, e somente se, $\|\varphi \rightarrow \psi\|=1$. Demonstração: Imediata da definição acima.

Corolário 6.20: Para $\varphi, \psi \in \operatorname{For}(\mathrm{L}),\|\varphi\| \nrightarrow\|\psi\|=1$ se, e somente se, $\|\varphi\| \subseteq\|\psi\|$.

Demonstração: $\|\varphi\| \rightarrow\|\psi\|=1 \Leftrightarrow\|\varphi \rightarrow \psi\|=1 \Leftrightarrow \vdash \varphi \rightarrow \psi \Leftrightarrow\|\varphi\| \subseteq\|\psi\|$. . 
Teorema 6.21: A álgebra $A_{\|}=(\{\|\varphi\|: \varphi \in \operatorname{For}(\mathrm{L})\}, \rightarrow, 1)$ é implicacional.

Demonstração: (i) $\|\varphi\| \rightarrow 1=1$, pois $\vdash \varphi \rightarrow(\varphi \rightarrow \varphi)$.

(ii) Se $\|\varphi\| \mapsto\|\psi\|=1$ e $\|\psi\| \rightarrow\|\varphi\|=1$, então $\|\varphi\| \subseteq\|\psi\|$ e $\|\psi\| \subseteq$ $\|\varphi\|$. Logo $\|\varphi\|=\|\psi\|$.

(iii) Como $\vdash \varphi \rightarrow(\psi \rightarrow \varphi)$, então $\|\varphi\| \subseteq\|\psi \rightarrow \varphi\|=\|\varphi\| \nrightarrow\|\psi\|$. Do corolário anterior, $\|\varphi\| \mapsto(\|\varphi\| \mapsto\|\psi\|)=1$.

(iv) Como $\vdash(\varphi \rightarrow(\psi \rightarrow \sigma)) \rightarrow((\varphi \rightarrow \psi) \rightarrow(\varphi \rightarrow \sigma))$, por várias aplicações do corolário anterior temos $(\|\varphi\| \rightarrow(\|\psi\| \rightarrow\|\sigma\|)) \rightarrow(($ $\|\varphi\| \rightarrow\|\psi\|) \rightarrow(\|\varphi\| \rightarrow\|\sigma\|))=1$.

Proposição 6.22: Cada teoria $\Delta$ de iL é um filtro implicativo para $\varphi$ $\leq \psi \Leftrightarrow \vdash \varphi \rightarrow \psi$.

Demonstração: Certamente, $\varphi \rightarrow \varphi$ pertence a toda teoria $\Delta$ de iL, e se $\varphi \in \Delta$ e $\varphi \leq \psi$, então $\varphi \rightarrow \psi \in \Delta$ e, daí, por MP, $\psi \in \Delta$.

Teorema 6.23: (Completude Forte) Seja $\Gamma \cup\{\varphi\} \subseteq$ For $(L)$. Se $\Gamma \vDash \varphi$, então $\Gamma \vdash \varphi$.

Demonstração: Consideremos que $\Gamma H \varphi$ e $\Delta$ é o fecho dedutivo de $\Gamma$. Então $\Delta$ é uma teoria tal que $\varphi \notin \Delta$. Pela proposição anterior, $\Delta$ é um filtro implicativo de iL e das Proposições 4.17 e 4.19, temos que existe um filtro maximal $\Delta^{*}$ tal que $\Delta \subseteq \Delta^{*}$ e $\varphi \notin \Delta *$. Logo, $\Delta * \in \mathbf{T}$. Assim, há um modelo $\Delta *$ em uma iálgebra, a álgebra $A_{\|}$, tal que $\Delta^{*} \vDash \Gamma$ e $\Delta^{*} \not \models \varphi$. Logo, $\Gamma \not \varphi$.

\section{Uma álgebra implicacional a partir dos espaços de Tarski}

Estabeleceremos agora uma conexão entre alguns dos conceitos tratados neste artigo, a saber veremos como obter uma álgebra implicacional a partir dos espaços de Tarski, um espaço conjuntista, que põe em evidência a noção de consequência e também de ordem. 
Vimos, nas Definições 3.8 e 3.9, as noções de Espaço de Tarski (E, $\mathcal{C}$ ), e de conjunto fechado em um Espaço de Tarski. Em uma primeira abordagem tentando obter uma iálgebra a partir de um Espaço de Tarski consideramos a reunião de todos os fechados do espaço e adotamos o seguinte procedimento.

Seja $(E, \mathcal{C})$ um espaço de Tarski e $\mathcal{F}$ e a coleção de todos os conjuntos fechados desse espaço, isto é, $\mathcal{F}=\{\mathrm{X} \subseteq \mathrm{E}: \mathcal{C}(\mathrm{X})=\mathrm{X}\}$.O conjunto $\mathcal{F}$ tem como elemento máximo o conjunto $E$ e como elemento mínimo o conjunto $\mathcal{C}(\varnothing)$.

Definição 7.1: Para quaisquer $X, Y \in \mathcal{F}$, definimos uma operação binária, denotada por $m$, do seguinte modo:

$$
X \backsim Y=\mathcal{C}\left(X^{\subset} \cup Y\right) \text {. }
$$

O conjunto $X$ é fechado, mas o seu complementar $X^{\complement}$ não precisa ser fechado, nem tampouco o conjunto $X \subset \cup Y$. Mas o seu fecho certamente é fechado.

Conforme será visto na próxima proposição, a estrutura $(\mathcal{F}, \rightsquigarrow, \mathrm{E})$ se aproxima de uma iálgebra.

Proposição 7.2: Na estrutura ( $\mathcal{F}, \rightsquigarrow$, E), acima definida, são válidas:

(i) $X \leadsto E=E$;

(ii) se $X \backsim Y=E$ e $Y \rightsquigarrow X=E$, então $X=Y$;

(iii) $X \rightsquigarrow(Y \rightsquigarrow X)=E$

Demonstração: (i) $\mathrm{X} \leadsto \mathrm{E}=\mathcal{C}\left(\mathrm{X}^{\mathrm{C}} \cup \mathrm{E}\right)=\mathcal{C}(\mathrm{E})=\mathrm{E}$.

(ii) Como o operador identidade é um operador de Tarski sobre $E$, para os seus fechados $\mathcal{F}$ temos o seguinte: se $X \neq Y$, então $X^{c} \cup Y \neq E$ ou $Y^{C} \cup X \neq E$.

(iii) Segue pela Proposição 3.13, que: $X \leadsto(Y \leadsto X)=X \rightsquigarrow \mathcal{C}\left(Y^{c} \cup X\right)=$ $=\mathcal{C}\left(X^{\subset} \cup \mathcal{C}\left(Y^{\subset} \cup X\right)\right)=\mathcal{C}\left(X^{\subset} \cup Y^{c} \cup X\right)=\mathcal{C}(E)=E$.

Apesar dos resultados acima, não conseguimos validar a condição $\left(a_{4}\right)$, da Definição 4.1, na estrutura ( $\mathcal{F}, \rightsquigarrow$, E) proposta. Contudo, conseguimos 
contornar a deficiência ao tomarmos um subconjunto muito especial de $\mathcal{F}$ ,que reúne os seus elementos que são simultaneamente abertos e fechados.

Proposição 7.3: Em todo espaço de Tarski $(E, \mathcal{C})$, tem-se $[\mathcal{C}(X)]^{c} \subseteq \mathcal{C}\left(X^{C}\right)$. Demonstração: Como $\mathrm{X} \subseteq \mathcal{C}(\mathrm{X})$, então $[\mathcal{C}(\mathrm{X})]^{\mathrm{c}} \subseteq \mathrm{X}^{\mathrm{C}} \subseteq \mathcal{C}\left(\mathrm{X}^{\mathrm{C}}\right)$.

Definição 7.4: Em um espaço de Tarski $(E, \mathcal{C})$, um conjunto $X \subseteq E$ é denominado clopen se ele é aberto e fechado simultaneamente.

Denotamos o conjunto de todos os clopens de $(E, \mathcal{C})$ por $G$.

Proposição 7.5: Seja $(E, \mathcal{C})$ um espaço de Tarski. Se $\mathcal{S} \subseteq \mathcal{P}(E)$ é tal que, para todo $\mathrm{X} \in \mathcal{S}$, tem-se $[\mathcal{C}(\mathrm{X})]^{\mathrm{c}}=\mathcal{C}\left(\mathrm{X}^{\mathrm{C}}\right)$, então $\mathcal{S} \subseteq G$.

Demonstração: Cada fechado de $(E, \mathcal{C})$ é do tipo $\mathcal{C}(X)$, para algum $\mathrm{X} \subseteq \mathrm{E}$. Daí, $[\mathcal{C}(\mathrm{X})]^{\mathrm{C}}$ é aberto e como $[\mathcal{C}(\mathrm{X})]^{\mathrm{c}}=\mathcal{C}\left(\mathrm{X}^{\mathrm{c}}\right)$, então ele é aberto e fechado. Cada aberto de $(E, \mathcal{C})$ é do tipo $[\mathcal{C}(X)]^{c}$, para algum $X \subseteq \mathrm{E}$. Como $[\mathcal{C}(X)]^{c}=\mathcal{C}\left(X^{c}\right)$, então ele é aberto e fechado.

Como consequência, no espaço de $\operatorname{Tarski}(G, \mathcal{C})$, temos que $[\mathcal{C}(E)]$ ${ }^{c}=[\mathrm{E}]^{\mathrm{C}}=\varnothing$ e, então, $\mathcal{C}\left(\mathrm{E}^{\mathrm{C}}\right)=\mathcal{C}(\varnothing)=\varnothing$. Esse fato acarreta que $(G, \mathcal{C})$ é um espaço vácuo.

Além disto, $\mathcal{C}\left(\varnothing^{\mathrm{c}}\right)=\mathcal{C}(\mathrm{E})=\mathrm{E}$ e $[\mathcal{C}(\varnothing)]^{\mathrm{c}}=[\varnothing]^{\mathrm{C}}=\mathrm{E}$.

Proposição 7.6: Seja $(E, \mathcal{C})$ um espaço de Tarski e $(G, \mathcal{C}$ ) o seu subespaço de clopens. Então a estrutura $(G, \rightsquigarrow, E)$, tal que para $X, Y \in G$, definimos $X \leadsto Y=\mathcal{C}\left(X^{\complement} \cup Y\right)$, é uma iálgebra.

Demonstração: Tendo em vista a Proposição 7.2 e o fato de que $G$ $\subseteq \mathcal{F}$, resta-nos mostrar que vale a condição $\left(\mathrm{a}_{4}\right)$ da Definição 4.1 , isto é, para todos $X, Y \in G$ : 


$$
(X \rightsquigarrow(Y \rightsquigarrow Z)) \rightsquigarrow((X \rightsquigarrow Y) \rightsquigarrow(X \rightsquigarrow Z))=1 .
$$

Para isso, observemos que, $X \backsim(Y \backsim Z)=X \backsim\left(\mathcal{C}\left(Y^{c} \cup Z\right)\right)=\mathcal{C}\left(X^{c}\right.$ $\left.\cup \mathcal{C}\left(Y^{c} \cup Z\right)\right)=\mathcal{C}\left(X^{c} \cup Y^{c} \cup Z\right)$.

Por outro lado, usando as Proposições 3.13 e 7.5, obtemos:

$$
(X \leadsto Y) \cdots(X \rightsquigarrow Z)=\mathcal{C}\left(X^{\subset} \cup Y\right) \cdots \mathcal{C}\left(X^{c} \cup Z\right)=\mathcal{C}\left([ \mathcal { C } ( X ^ { c } \cup Y ) ] ^ { c } \cup \mathcal { C } \left(X^{c}\right.\right.
$$

$\cup Z))=\mathcal{C}\left(\left[\mathcal{C}\left(X^{c} \cup Y\right)\right]^{c} \cup\left(X^{c} \cup Z\right)\right)=\mathcal{C}\left(\mathcal{C}\left[\left(X^{C} \cup Y\right)^{c}\right] \cup\left(X^{c} \cup Z\right)\right)=\mathcal{C}\left(\left(X^{c}\right.\right.$ $\left.\cup Y)^{\mathcal{C}} \cup\left(X^{\subset} \cup Z\right)\right)=\mathcal{C}\left(\left(X \cap Y^{c}\right) \cup\left(X^{\subset} \cup Z\right)\right)=\mathcal{C}\left(\left(X \cup X^{c} \cup Z\right) \cap\left(X^{c} \cup Y^{c}\right.\right.$ $\cup Z))=\mathcal{C}\left(E \cap\left(X^{C} \cup Y^{C} \cup Z\right)\right)=\mathcal{C}\left(X^{C} \cup Y^{C} \cup Z\right)$.

Como os dois termos são iguais, então vale $\left(a_{4}\right)$ e, assim, $(G, \cdots, E)$ é uma iálgebra.

\section{Considerações finais}

Nestas notas, tratamos das noções de consequência lógica e ordem, primeiro em sistemas independentes que procuram formalizar os conceitos, e depois com uma lógica implicacional que ressalta o operador de implicação e internaliza o 'se, então' em um sistema formal específico dado por esta lógica.

Em todos os sistemas tratados, percebemos que a noção de ordem se faz presente. A lógica implicacional pediu um pouco mais que a ordem. Os axiomas da lógica implicacional conduzem à ordem, mas só a ordem não dá conta do sistema tratado. Parece razoável admitirmos que lógica exige um sistema de ordem e algo mais. Na lógica tratada, este algo mais é explicitado pelos seus axiomas e correlatos da iálgebra.

Como mais uma construção, obtivemos um caso bem geral, que acreditamos ser um teorema de representação das iálgebras, dado pelos espaços de clopens de qualquer espaço de Tarski.

Em momentos posteriores, devemos continuar a tratar deste conceito basilar que é a ordem na inter-relação com a Lógica. Vemos aqui aspectos dos fundamentos da Lógica e uma visão bastante geral a ser tratada. 


\section{Referências}

FEITOSA, H. A.; LAZARO, C.A.; NASCIMENTO, M. C. Pares de Galois e espaços de Tarski. Cognitio: Revista de Filosofia, São Paulo, v. 19, n. 1, p. 110-132, 2018. https://doi.org/10.23925/2316-5278.2018v19i1p110-132

FEITOSA, H. A.; NASCIMENTO, M. C.; ALFONSO, A. B. Teoria dos conjuntos: sobre a fundamentação matemática e a construção dos conjuntos numéricos. Rio de Janeiro: Ciência Moderna, 2011.

FEITOSA, H. A.; PAULOVICH, L. Um prelúdio à lógica. São Paulo: Editora UNESP, 2005.

FEITOSA, H. A.; MOREIRA, A. P. R.; SOARES, M. R. Operadores de consequência e relações de consequência. Kínesis, Marília, SP, v. 8, n. 18, p. 156-172, 2016. FEITOSA, H. A.; MOREIRA, A. P. R.; SOARES, M. R. Sobre relações de consequência com múltiplas conclusões. Cognitio: Revista de Filosofia, São Paulo, v. 17, n. 1, p. 73-82, 2016.

MARTIN, N. M.; POLLARD, S. Closure spaces and logic. Dordrecht: Kluwer, 1996. https://doi.org/10.1007/978-1-4757-2506-3

RASIOWA, H. An algebraic approach to non-classical logics. Amsterdam: North-Holland, 1974.

\section{Endereço postal}

Unesp - Faculdade de Ciências- Campus de Bauru

Av. Eng. Luiz Edmundo Carrijo Coube, 14-01

Vargem Limpa - Bauru-SP

CEP: $17033-360$ 